\title{
TU/e emonownen

\section{Suboptimal bonding impairs hormonal, epigenetic and neuronal development in preterm infants, but these impairments can be reversed}

\section{Citation for published version (APA):}

Kommers, D. R., Oei, S. G., Chen, W., Feijs, L. M. G., \& Bambang Oetomo, S. (2016). Suboptimal bonding impairs hormonal, epigenetic and neuronal development in preterm infants, but these impairments can be reversed. Acta Paediatrica, 105(7), 738-751. https://doi.org/10.1111/apa.13254

DOI:

10.1111/apa.13254

Document status and date:

Published: 01/07/2016

\section{Document Version:}

Accepted manuscript including changes made at the peer-review stage

\section{Please check the document version of this publication:}

- A submitted manuscript is the version of the article upon submission and before peer-review. There can be important differences between the submitted version and the official published version of record. People interested in the research are advised to contact the author for the final version of the publication, or visit the $\mathrm{DOI}$ to the publisher's website.

- The final author version and the galley proof are versions of the publication after peer review.

- The final published version features the final layout of the paper including the volume, issue and page numbers.

Link to publication

\footnotetext{
General rights

- You may freely distribute the URL identifying the publication in the public portal. follow below link for the End User Agreement:

www.tue.nl/taverne

\section{Take down policy}

If you believe that this document breaches copyright please contact us at:

openaccess@tue.nl

providing details and we will investigate your claim.
}

Copyright and moral rights for the publications made accessible in the public portal are retained by the authors and/or other copyright owners and it is a condition of accessing publications that users recognise and abide by the legal requirements associated with these rights.

- Users may download and print one copy of any publication from the public portal for the purpose of private study or research.

- You may not further distribute the material or use it for any profit-making activity or commercial gain

If the publication is distributed under the terms of Article $25 \mathrm{fa}$ of the Dutch Copyright Act, indicated by the "Taverne" license above, please 


\title{
Suboptimal bonding impairs hormonal, epigenetic and neuronal development in preterm infants, but these impairments can be reversed
}

\author{
D Kommers (Deedee.Kommers@mmc.nl) ${ }^{1,2}$, G Oei ${ }^{3,4}$, W Chen ${ }^{2}$, L Feijs ${ }^{2}$, S Bambang Oetomo ${ }^{1,2}$ \\ 1.Department of Neonatology, Máxima Medical Centre Veldhoven, Veldhoven, The Netherlands \\ 2.Department of Industrial Design, Eindhoven University of Technology, Eindhoven, The Netherlands \\ 3.Department of Electrical Engineering, Eindhoven University of Technology, Eindhoven,The Netherlands \\ 4.Department of Gynaecology, Máxima Medical Centre Veldhoven, Veldhoven,The Netherlands
}

\author{
Keywords \\ Bonding, Development, Neonatal intensive care \\ unit, Parent, Preterm infant

\section{Correspondence} \\ D Kommers, Department of Neonatology, Máxima \\ Medical Centre, De Run 4600, 5504 DB, \\ Veldhoven, The Netherlands. \\ Tel: +31 648700297 | \\ Fax: +31408889340 | \\ Email: Deedee.Kommers@mmc.nl \\ Received \\ 26 March 2015; revised 24 June 2015 ; \\ accepted 26 October 2015.
}

\begin{abstract}
This review aimed to raise awareness of the consequences of suboptimal bonding caused by prematurity. In addition to hypoxia-ischaemia, infection and malnutrition, suboptimal bonding is one of the many unnatural stimuli that preterm infants are exposed to, compromising their physiological development. However, the physiological consequences of suboptimal bonding are less frequently addressed in the literature than those of other threatening unnatural stimuli.

Conclusion: This review found that suboptimal bonding significantly impaired hormonal, epigenetic and neuronal development, but these impairments could be reversed by bonding interventions. This suggests that neonatal intensive care units should focus more on interventions that optimise bonding.
\end{abstract}

DOI:10.1111/apa.13254

\section{INTRODUCTION}

Over the past 20 years, the incidence of preterm birth has increased and stabilised at an estimated $12 \%$ in the USA and $4-8 \%$ in high-income countries in Europe (1). Both the mortality rate and the incidence of major disabilities, such as cerebral palsy and mental retardation, have decreased due to improvements in perinatal health care (2). However, the long-term morbidity of these infants is still a serious health concern (1). Minor morbidities such as attention deficits, academic underachievement and hyperkinetic disorders occur frequently $(1,2)$. Marcos et al. stated that both major and minor morbidities are probably induced by the fact that a preterm infant is exposed to unnatural stimuli that it would not be exposed to in the uterus (1). Indeed, it has been widely established that unnatural stimuli such as painful procedures, impaired nutrition and medical conditions such as hypoxia-ischaemia and infection can disturb homeostasis and hence normal organ development (3). Development might also be influenced by another unnatural stimulus that is an inevitable consequence of premature birth and that is altered parent-infant bonding (4).

In the medical literature, developmental consequences of altered parent-infant bonding are overshadowed by the

\footnotetext{
Abbreviations

BDNF, Brain-derived neurotrophic factor; EEG, Electro-encephalogram; HPA, Hypothalamic-pituitary-adrenal; KC, Kangaroo care; mRNA, Messenger-RNA; NICU, Neonatal intensive care unit; NMDA, N-methyl-D-aspartate.
}

consequences of many other unnatural stimuli. This could be explained by the fact that there is no objectively measurable physiological parameter for the degree of bonding. This creates a challenge for directly addressing the research question about whether suboptimal bonding alters development. On the other hand, bonding-related perinatal aspects, or in other words elements of bonding, have been studied separately. For example, there is an abundance of literature on the influence of perinatal maternal separation available (5), as well as literature on the physiological effects of the amount of perinatal exposure to licking and grooming. Licking and grooming is the animal analogue of caressing (6). In this review, we try to answer the previously formulated research question about whether suboptimal bonding alters development as accu-

\section{Key notes}

- In addition to hypoxia-ischaemia, infection and malnutrition, suboptimal bonding is one of the many unnatural stimuli that preterm infants are exposed to.

- The physiological consequences of suboptimal bonding are less frequently addressed in the literature than those of other threatening unnatural stimuli.

- This review found that suboptimal bonding significantly impaired hormonal, epigenetic and neuronal development, but these impairments could be reversed by bonding interventions investigated at neonatal intensive care units. 
rately as possible, by bringing the analysed, separate influences of various bonding-related perinatal factors together. To the best of our knowledge, this has never been made before. To be able to do this, it is necessary to define bonding. What makes maternal licking, grooming and separation bonding-related factors?

Two of the founders of the definition of bonding were Bowlby and Ainsworth. The developmental history of their attachment theory began in the 1930s with Bowlby's interest in the link between social deprivation distress and later personality development. He revolutionised the way of thinking about a child's attachment to the mother and the disruption that was caused by separation (7). His theory stated that the crying and clinging behaviour that infants demonstrated to prevent being separated from their parents was evolutionary based. These behaviours are even more important when it comes to increasing the chance of survival than sucking behaviours (7). An infant needs to develop a relationship with his or her primary caregiver in order to survive and these behaviours more or less force that relationship. Such behaviours can thus be termed bonding-related factors. In addition to describing the cause of infant behaviours, Bowlby and Ainsworth used infant behaviours and parental behaviours in certain situations to classify those relationships (7). For instance, sensitive mothers were likely to display comforting and protecting behaviour in threatening situations (7). Behaviour in certain situations, such as the licking and grooming behaviour of rats, can thus be seen as bonding related. Infants from sensitive mothers are most likely to display sociable, exploratory behaviour. According to the attachment theory, this is because sensitive mothering frequently leads to infants being securely attached, which is one example of a relationship classification within the attachment theory (7). These relationship classifications and the attachment theory have been very important to bonding-related research. However, there has been some constructive criticism on the attachment theory.

The theory is limited with regard to providing objectively measurable study inputs and outputs. Observations and measurements of behaviours are open to multiple interpretations, family characteristics are complex, and numerous variables can confound study results (8). Observations are a very useful tool for clinicians, as they focus mainly on the end of the spectrum to diagnose attachment disorders. However, for researchers who are more interested in the subtleties of the spectrum, ongoing questions about these observational measures have been barriers to large-scale studies (8). Furthermore, even though the attachment theory has been partially based on animal studies, it is a theory restricted to human attachment. Regarding the fact that parallels can be drawn between the maternal and infant behaviours of different species and that the attachment behaviours of all species appear to influence homeostasis, the restriction to the human species appears to be a limitation (8). This limitation and the finding that attachment behaviours influence homeostasis have been incorporated into the current definition of bonding by psychobiologists and neuropsychologists. According to them, bonding should be described as the process of coregulation, when an individual uses his or her capacity to regulate the internal environment of others as well as themselves $(9,10)$.

In medical terms, such regulation of one's internal environment is called homeostasis. In order to be able to regulate not only one's own, but also another's internal environment, the other's internal environment must be read. An organism's internal environment is expressed by all of its physical parameters, or cues, such as heart rate, temperature, scent, skin colour, voice and facial expression. Every organism has been given appropriate sensitivities to pick up and respond to cues from those other organisms to whom bonding could be beneficial for survival (11). Humans are extremely good at picking up cues and responding to them, due to their uniquely large cortex. This quality has resulted in a very complex social system with enormously diverse, intricate bonds (12). When a human being either unconsciously or consciously reads another's internal state and perceives it as stressed, he or she can act upon that by providing comfort, for instance through touching, feeding or giving shelter. This then contributes to the regulation of the internal state of the other person. This capacity to form bonds through cues and sensitivities to those cues is controlled by a complex physiological mechanism, built in during evolution, in order to maximise the species' chances of survival $(11,12)$.

Unfortunately, prematurity alters these cues and sensitivities and, therefore, bonding, in many ways. Preterm infants' cues are scarce and often unrecognisable as, due to their physical immaturity, they are not capable of expressing their internal states in the way that term infants can (10). Furthermore, the need to be admitted to a neonatal intensive care unit (NICU) also contributes to impaired bonding. The tubes, patches, noises, bright lights, incubators and painful procedures that come with a NICU stay reduce the quality and quantity of both cues and the sensitivity to cues from the mother and child $(4,13)$. Tubes and patches impede body expressions. Incubators are physical barriers to comforting and touch, and they separate the child from his or her parents and so on. Thus, preterm birth disturbs coregulation of the internal environment, bonding, in a period of time when this actually is needed more than usual for both the infant and parent $(9,14)$. Consequently, in addition to medical conditions such as hypoxia-ischaemia and infection, suboptimal bonding probably contributes to developmental impairments in prematurely born infants. This study was therefore undertaken to place the emphasis on the consequences of suboptimal bonding and to consecutively raise the awareness of clinicians about this important matter.

\section{METHODS}

For this semi-systematic literature review, a MEDLINE and EMBASE search was performed from May until November 2014. This paragraph provides a stepwise version of the 
subsequent phases in the process. For a more detailed methods section, see Appendix.

In phase one, bonding-related terms were extracted from the available literature. In phase two, the available literature was divided into categories. According to the article's relevance, only the title, title and abstract, or title, abstract and full text were scanned to exclude, include or redistribute articles into categories. Predetermined criteria were used for this. In phase three, an extensive, nonselective work containing all the findings from all the included articles for each topic was created. Then, the review was narrowed down by either including or excluding findings, using predetermined criteria. During the subsequent revision phase, the search was repeated so that it was as up to date as possible. For the search terms, categories, and predetermined criteria, see Tables A1 - A5. For a flowchart of the literature that was included, see Figure A1.

\section{RESULTS}

We extracted 23 bonding-related perinatal factors from the literature, including the following: licking, grooming, presence or absence (separation), feeding routines, sensitivity, received care, tactile stimulation and body heat. However, many papers used more general terms to capture bondingrelated perinatal factors, such as early life experiences, early life adversity, NICU stay or NICU admission.

\section{NICU-related early life experiences that altered physiology}

NICU-related early life experiences were reviewed in a booklet edited by Browne and White called Clinics in Perinatology - Foundations in Developmental Care (13). They stated that, in the light of the remarkable plasticity during early development, the significant modifications of sensory experience that come with preterm birth and a necessary NICU admission are likely to have a range of effects on the normal course of development'. However, they also stated that 'investigators are a long way from understanding the particulars'. Another key point made in the booklet was that, compared with their term-born peers, preterm children have a much less developed behavioural repertoire, because of the immaturity of their brains. Important differences between the brains of term infants and preterm infants were highlighted by Browne et al. For example, brain volumes and brain region volumes are significantly different. Furthermore, the protein brainderived neurotrophic factor (BDNF) appears to be lower in preterm infants. BDNF is a protein that supports neuronal survival and encourages the growth of new neurons. It thereby stimulates brain development and brain plasticity (13). Additionally, a significantly reduced capacity for, and duration of, long-term depression neuroplasticity has been found in preterm infants (15). Long-term depression is an immediate reduction in the efficacy of neuronal synapses after activation of those synapses. It plays an important role in the activity-dependent pruning or reinforcing changes of neuronal development. This means that when neurons fire frequently, their pathways are reinforced, for instance, their myelin sheets will thicken. However, when firing in certain neuronal pathways is scarce, neuronal apoptosis will follow (15). Research needs to be extended in order to investigate whether long-term potentiation neuroplasticity, the opposite of long-term depression neuroplasticity, is also reduced (13). Either way, whether or not that is the case, the demonstrated physiological differences in preterm infants make them extremely vulnerable. The fact that the physiological differences we report here are only some of the many known physiological differences caused by being born prematurely makes them even more vulnerable (13).

This instant presence of physiological differences due to premature birth is relevant to the research question about whether suboptimal bonding alters development. Apparently, preterm infants are already more vulnerable prior to the exposure to any additional unnatural stimulus, including unnatural bonding experiences. This was both a limitation and a strength of this review. It was a limitation because it impeded the assessment of the isolated effects of exposure to unnatural stimuli. On the other hand, it was a strength, because it suggested that, due to their vulnerability, the effects of all unnatural stimuli, including suboptimal bonding, could have major effects and they should therefore receive clinical attention.

In her review, Grunau (3) also drew attention to effects of certain unnatural stimuli. She reported on the long-term effects of stress caused by a NICU admission, specifically procedural pain-related stress. Grunau stated that 'procedural pain and stress in preterm infants are associated with abnormal brain development in the NICU, above and beyond other clinical risk factors'. Grunau was also part of the research team that found that greater neonatal pain and stress exposure, measured by the number of skin breaking procedures, was associated with slower body and head growth. This was associated with altered cortical grey matter on diffusion tensor imaging at term. Greater exposure to procedural pain-related stress was associated with reduced development of white matter and subcortical grey matter (16). Furthermore, Grunau pointed out a longitudinal study from Doesburg et al. In that study, cumulative neonatal pain exposure in infants that were born prematurely was associated with changes in background cortical rhythmicity on magneto-encephalography measured later in childhood. The spectral structure of cortical oscillations expressed in power ratios among oscillations in different frequency ranges was assessed. Corrected for potential confounders, the found alterations in such spontaneous brain oscillations remained negatively correlated with visual-perceptual abilities at school age (17).

Anand et al. also reviewed the literature on alterations in adult brains due to repetitive neonatal pain and stress experiences. They focused more on maternal separation stress and the mechanisms that caused the effects of pain and stress. They proposed a two-way mechanism: hypoactivation of N-methyl-D-aspartate (NMDA) receptors is caused by maternal separation and sensory isolation, which 
leads to increased apoptosis in multiple areas of the immature brain, while at the same time hyperactivation of NMDA receptors in other brain areas is caused by an exposure to repetitive pain (18). According to Anand et al., the latter results in excitotoxic damage to developing neurons. NMDA receptors are a subtype receptor for the most dominant excitatory transmitter system in the brain, the glutamate system. Thereby, NMDA receptors play a central role in the activity-dependent pruning or reinforcing of neurons (18). The complexity of the mechanism by which pain causes neuronal damage is not fully understood, but the fact that repetitive neonatal pain is harmful, and that there is involvement of the hypothesised mechanisms, is supported by various later studies $(19,20)$. Furthermore, recent electro-encephalogram (EEG) findings suggest that the preterm neonatal brain is more sensitive to pain and that preterm infants are not very capable of distinguishing tactile from nociceptive stimulation, creating even more complexity for the preterm infant's caretakers (3). The infant's immaturity in mimicry, day and night rhythm and behavioural repertoire also imposes on the infant's readiness to enter the caregiving dialogue, while on the parental side fear and the hindrance of the tubes, patches and the incubator disturb the caregiving dialogue (13). Programmes designed to recognise infant cues and to provide supportive care have, therefore, developed over the past decade, such as the Newborn Individualised Developmental Care and Assessment Program (NIDCAP) mainly established by Als.

The concept of developmental care is a framework for NICU staff that ensures that each individual infant is as stable, well organised and competent as possible (14). The infant's own physiological and behavioural expressions are professionally assessed and seen as a reliable guide for caregivers to act upon. The family is seen as the primary caregiver and thus stimulated to be maximally involved in the caregiving (14). In a Cochrane review that included 627 preterm infants, however, no significant evidence was found that NIDCAP improved long-term neurodevelopmental or short-term medical outcomes (21). This is remarkable, as there is converging evidence that preterm infants are more developmentally vulnerable to parentinfant interaction, suggesting that the parents' behaviours play a key role in their child's neurodevelopment (3). Maximum parental involvement, for instance, due to a programme such as NIDCAP, should compensate for adverse clinical exposure and compromised early brain development $(3,13)$. In some of the trials included in the Cochrane review such compensating effects were indeed found. Whereas the primary outcomes of reduction of mortality and severe disabilities showed no significant differences, shorter hospital stays, growth benefits and improved scores on neurodevelopmental scales at nine months of corrected age were reported (21). Nonetheless, based on all of the results and its cost-effectiveness, Ohlsson et al. concluded that no clear benefits of NIDCAP could be identified. The implementation of NIDCAP in its present form was therefore not recommended. However, Ohlsson et al. did recommend maximum involvement of the parents in caregiving, as they believed in the benefits of this (22).

This was supported by a cohort study by Grunau et al. that demonstrated that greater positive maternal interaction during painful procedures buffered the relationship between neonatal procedural pain exposure and poorer focused attention in very preterm infants at eight months of corrected age. More maternal interaction was also protective against infants' internalising anxiety or depressive behaviours at 18 months of corrected age (3). So perhaps, compared to the current standard care in NICUs, NIDCAP is not enriching enough to lead to significant results. Over the past decades, even without having a programme such as NIDCAP implemented, most NICUs started focusing on individualised caregiving and also on involving the parents (23). A more easily measurable factor for caregiving enrichment would be an increase in parents visiting the NICU. Only very few studies have looked at this. They identified infrequent maternal visits as a risk factor for later psychological development in preterm infants (24). The above implies that both bonding-related and nonbonding-related early life experiences affect physiological development in a context-dependent way. Painful stimuli, maternal separation and abuse can be excitotoxic for neuronal development, whereas adequate caregiving can be protective.

Such a parental protection hypothesis is significantly supported by previous work from Sullivan's research group. They addressed the question of why a child became attached to caregivers regardless of the quality of care they received. The key point that Sullivan stated was that the child's brain was not an immature version of the adult brain. Its circuitry was designed to ensure attachment to caregivers. As proximity to a caregiver is necessary in order to survive, a child should therefore attach to any caregiver (25). In their studies, attachments to abusive caregivers resulted in dysregulation in neural networks, especially in dysregulated amygdala function. The amygdala is a brain area within the limbic system, which is required for fear and avoidance learning in adult animals. By carefully manipulating the abuse-related attachment learning in rat pups, Sullivan et al. discovered that during a sensitive period early in life, the activation of the amygdala was blocked whenever there was maternal presence. Parental abuse did therefore not cause a fear and learn to avoid reaction when an infant's mother was present. In their laboratory, pups formed attachments even when painful abuse was experienced. This infant experience resulted in later life depressive-like behaviour and altered amygdalas with suboptimal connectivity to the prefrontal cortex. Experiencing nonabusive painful procedures on the other hand did not result in amygdala changes, indicating that during attachment learning, pain was processed differently (25). More knowledge of this would be extremely valuable for NICUs, because even though painful experiences are inevitable, the negative effects of these might be minimised by high-quality parental caregiving. Moreover, the differences in physiological parameters due to prematurity itself are difficult to treat, 
but optimising early life experiences might be easier. Indeed, noninvasive interventions targeted at parental care are relatively easy to implement, according to Walker et al. Moreover, these interventions might have a significant effect on the health outcome of the offspring, particularly in a vulnerable population of preterm babies (26).

Over the past few decades, researchers in the Braun laboratory have attempted to provide evidence for this parental protection theory. In one study, they used a maternal separation paradigm on the Octodon Degus trumpet-tailed rat species and investigated whether a mother's voice during maternal separation could protect from separation-induced changes of brain function. Compared to the control group, the separation group showed an upregulation of NMDA receptor density in the prefrontal cortex, hippocampus and amygdala. In the third group, presentation of the maternal call during the separation period suppressed the NMDA receptor upregulation in all brain regions (27). In a 2014 review, Bock et al. (28) summarised their work, and the work of others, to provide an integrative view on this topic. They reported that 'evidence is now accumulating that the specific and individual effects of early life experiences on the functional development of brain and behaviour emerge as a function of type, intensity, timing and the duration of the events'. In their studies, the effect of separation seemed to be delayed pruning or, in other words, a disturbance in the guidance for the neuronal cells to optimally specialise (28). However, the most frequently reported physiological disturbances in other studies examining early life experiences are those related to the development of the hypothalamic-pituitaryadrenal (HPA) axis, also known as the stress axis.

\section{Mother-infant-related early life experiences and stress axis regulation}

Due to the sudden transition from intra-uterine to extrauterine life, early life is a very stressful period. Without a protection mechanism, the ongoing stress and the constantly hyperactive HPA axis would lead to chronic glucocorticoid exposure, which would cause cellular damage (26). A mechanism to protect organisms against this damage can, therefore, also be described as an adequate regulatory mechanism. However, the newborn infant's own regulatory mechanisms are not sufficiently mature to accomplish this protection. The newborn infant needs coregulation for optimal protection and thus optimal development. Animal research has demonstrated that mothers are able to directly regulate the pup's homeostasis, including his or her cortisol levels, through hidden regulators (29). These regulators are for instance sensory, motor, nutrient and thermal stimuli embedded in typical motherinfant interactions, such as licking, grooming and archedback nursing. The parental influences on the infant's physiology often cannot be seen directly, but become evident in periods of stress or separation for instance demonstrated by fluctuations in blood pressure or hormonal concentrations (9). That is because the infant's stress regulatory system is, as previously stated, not yet mature enough to cope with separation or stress without parental support. The main axis within the elaborate stress regulatory system is the HPA axis, therefore also called the stress axis. Parent-infant interactions can thus exert an immediate coregulatory influence on the infant's HPA axis $(26,30)$. During the very stressful early life period, this coregulation results in suppressed cortisol activity, to protect the brain from otherwise constant glucocorticoid exposure. This period is called the stress hyporesponsive period.

During the stress hyporesponsive period, cortisol and the HPA axis are not the only systems that are affected. Other hormonal systems and, therefore, behavioural responses are affected in both the short-term and long-term. The immediate regulatory influence of typical mother-infant interactions is elaborate and necessary for optimal development $(26,30)$. Inadequate mother-infant interaction thus impairs optimal development. Long episodes of maternal separation, lasting 180 minutes, changed corticotropin-releasing factor signalling pathways and glucocorticoid receptors involved in negative feedback over the HPA axis in adult rats. Separated rats exhibited visceral hypersensitivity and were more prone to stress-induced intestinal mucosal dysfunction (5). Additionally, Walker et al. reported that inflammatory reactions were reduced in pups reared by their mothers compared to artificially reared pups (26).

Does this mean that the more care a mother provides, the better her offspring? The short answer is no. For example, a maximal maternal stimulation protocol used by Walker et al. showed higher pain sensitivity when compared to less maternal stimulation. This suggests that this protocol was beyond the optimal degree of stimulation provided by the mother. Too much maternal stimulation provided to a compromised brain, such as a premature brain, might be detrimental (26). Indeed, not only the presence, but also the sensitivity and responsiveness from caregivers during the early life period appeared critical in maintaining the desirable, low cortisol concentrations (6). To define optimal care giving, the relationship between the rats' HPA axis development and naturally occurring, individual differences in maternal care and thus maternal coregulation has frequently been evaluated (9). Liu et al. reported that the magnitude of the HPA response to stress in adult animals was strongly correlated with the degree of maternal licking and grooming of young pups (31). HPA axis alterations thus appeared context dependent. Indeed, both Rinaman et al. and Walker et al. reported that different lengths of maternal separation led to different HPA axis alterations $(5,26)$. In older pups and humans, social companionship instead of separation has also been demonstrated to influence HPA responses, a process called social buffering (32). Similar to maternal presence, this also works through coregulation, enabled by receiving multiple sensory cues from the social companion. However, the very early life social buffering system, the stress hyporesponsive period, has unique features. It is adapted specifically to the mother-infant dyad (32). According to Rincón-Cortés et al., maternal care and stressors early in life jointly programme HPA axis responses and functioning in later life (30). The question is 
no longer whether early life experiences influence the HPA axis, but exactly how they do it (33).

In an attempt to answer that question, Strüber et al. proposed a two-pathway model in which they addressed more hormones than just the stress axis hormones. With this model, they provided an explanation for why both the HPA axis hyperfunction and hypofunction were demonstrated in animal and human studies that evaluated the later life effects of different early life experiences (33). In their critical review, Tang et al. also advocated a more complex reality than the more the better. Their theory was called maternal modulation (6). According to the authors, the role of the mother is multidimensional, including not only maternal behaviour quantity, but also the quality of the maternal behaviour as demonstrated by reliability and sensitivity and the mother's capacity to regulate her own HPA function. Indeed, it is possible that infants who are fed by a stressed mother with a high cortisol concentration in her breastmilk have prematurely ended stress hyporesponsive periods (30). Interestingly, Tang et al. demonstrated, with results from animal and human studies, that the most influential factor for shaping key parameters of the infant's regulatory system, such as the HPA axis and other hormonal systems, was the caregiving prior to, and immediately after, exposure to a stressful stimulus. When offspring are stressed, it is the reliability, not the quantity, of maternal care that determines whether or not stressful novelty exposure will have a positive impact on the plasticity of the HPA axis during later life (6). Tang et al. concluded that high-quality parenting following negative life events could enhance positive adaptation during subsequent negative life events. This could also be true for highquality parenting following negative events during a NICU stay. From these results, it can be deducted that differences in maternal care and early life experiences set a cascade of physiological events in motion, impacting on the essence of human beings throughout the rest of their lives.

\section{Mother-infant-related early life experiences altering genetics}

Research has indeed demonstrated that the HPA axis is not the only physiological system that is altered by early life experiences and bonding-related experiences in particular. A growing body of neuroscience research has argued convincingly that an individual's early life experiences, or early life environment, together with an individual's genotype, influence his entire health and behavioural phenotype (34). Over the past few decades, an explanation for this has been discovered. Environmental factors can alter gene expression, without altering the genetic code itself. Instead, the frequency with which the genes are transcribed is altered. The transcription of the genome is regulated by intracellular molecules. The concentrations of these molecules are influenced by sensorial information about environmental factors. Together, those molecules and the genome itself are called the epigenome (35).

Epigenetic changes occur immediately after conception, providing a mechanism by which early caregiving environ- ments immediately have an impact on phenotypes, including the HPA axis phenotype (34). Letourneau et al. reported that negative early life experiences led to epigenetic modification (methylation) in the glucocorticoid receptor promoter, preventing binding with glucocorticoids. Such binding up regulates the production of anti-inflammatory proteins, which returns organisms to an unstressed state. Prevention of that binding decreases the facility with which the HPA axis can return to baseline after the threat of danger, leaving the organism in a state of heightened sensitivity to stress (34). In contrast, reduced sensitivity to stress has been reported in offspring of high licking and grooming dams when compared to offspring of low licking and grooming dams (36). This was demonstrated by increased hippocampal glucocorticoid receptor messenger-RNA (mRNA) expression, enhanced feedback sensitivity to glucocorticoids, decreased hypothalamic corticotropin-releasing factor mRNA expression and consequently more stable stress responses of the adrenocorticotropic hormone and of cortisol (37). Additionally, when infant pups of low licking and grooming mothers were cross-fostered to high licking and grooming mothers, glucocorticoid receptor mRNA expression and adrenocorticotropic hormone levels matched those of their high licking and grooming adopted mothers, not their low licking and grooming biological mothers. The inverse has also been demonstrated $(34,37)$. Kundakovic et al. (35) subscribed to the above, reporting that even when differences were within the normal range of parental behaviours, outcomes could be significantly influenced.

\section{Genetics, hormonal systems and neuronal development}

Murgatroyd et al. found reduced DNA methylation at one specific vasopressin gene region in the pariventricular nucleus (38). Vasopressin and oxytocin are hormones of special interest, because they appear to be key players for organisms' sociality and parental behaviours $(39,40)$. However, to enable a better analysis of epigenetic variations in the vasopressin or oxytocin genes, future studies using multiple gene marking and multiple area marking are necessary (39). Over the past few decades, various nongenetic study designs have demonstrated that the distribution and receptor systems of those hormones can be manipulated by early life experiences. Some of the designs were clinically very relevant, because they compared control subjects to subjects that were exposed to conditions mimicking a NICU environment, for instance using maternal separation and handling paradigms.

Todeschin et al. (41) found long-lasting changes when neonatal rat control subjects were compared to handled subjects. Handled pups were separated from their mothers and handled by the experimenter for one minute each day from postnatal day one to day 10 . Morphological changes in the neuroendocrine areas involved in social bonding in mammals were found, with oxytocin neurons being decreased and vasopressin neurons being increased in the pariventricular nucleus. In 2012, Veenema titled her review Toward understanding how early life social experiences 
alter oxytocin and vasopressin-regulated social behaviours (39). She incorporated seven different research paradigms in her review: maternal separation, early stress, single mothers, neonatal handling, communal nursing, high versus low licking and grooming and the manipulation 0 paradigm, a paradigm with no extra neonatal handling or manipulation, just weekly cage changes, while pups are transported in plastic cups. She concluded that all the paradigms had been shown to induce changes in the oxytocin and vasopressin systems. Therefore, those systems have shown a high level of plasticity as a function of the early life social environment. Currently, most authors deem that the question of whether or not early life experience has the ability to lead to long-term changes in oxytocin and vasopressin systems is undisputed. Instead, they consider the process and directionality by which experiences affect those and other systems $(39,40)$. Oxytocin systems are highly connected to for instance serotonin, noradrenaline and dopamine pathways.

Dopamine pathways are important for experiencing rewards. When neurohormonal circuitry functions adequately, sociality is experienced as rewarding. Studies have indicated that early life experiences have long-term programming effects on these pathways as well. In these studies, the long-term effects were defined as effects still present in adulthood (42). The direction of the changes was again complicated, as the influences were brain region related and often gender dependent (42). In addition to the dopamine system, influences of maternal care and early life experiences on the reproductive system have also been studied. These studies were reviewed by Toufexis et al. Their review demonstrated that the reproductive system was mainly investigated by examining differences in the functioning of the hypothalamic-pituitary-gonadal axis. Within this axis, alterations in the expression of sex hormone receptors such as the oestrogen receptor- $\alpha$ were reported (43). This hormone regulates sexual behaviour and the sexual strategy used by females. By influencing the sexual behaviour of the offspring, the effects from differences in maternal care become transgenerational (43).

Camozzato et al. (44) used a different approach to assess the hypothalamic-pituitary-gonadal axis. They explored the development of a brain area important to the control of ovulation, the medial pre-optic area. Using a neonatal handling paradigm, they found an approximate reduction percentage of $50 \%$ in the number of neuronal cells in that area at postnatal day 11 and still at day 90. A compensatory neuronal cell size increase was not found. On the contrary, the neonatal handling procedure decreased cell size. This study strengthened the conclusion of an early life environmental effect on the hypothalamicpituitary-gonadal axis by focusing on alterations in neuronal development.

\section{Mother-infant-related early life experiences that alter neuronal development}

Many studies have focused on altered neuronal development caused by different early life experiences. Some studies focused on specific brain areas, such as the medial pre-optic area. Other studies examined larger regions or even the entire brain, such as the study by Sarro et al. (45). They explored whether the cortical activity of rat pups was directly influenced by interactions with the mother. They recorded spontaneous neocortical potentials in freely behaving infant rats during natural interactions with their mother on postnatal days 12-19. Maternal absence increased cortical desynchrony. According to the authors, cortical desynchronisation was not necessarily negative, 'it is an activity pattern conducive to neural plasticity and information transfer across brain regions'. Removing littermates induced further desynchronisation. The authors stated that 'the results uncover that the mere presence of the mother provides an immediate impact on the state of cortical neural activity'. (45) The fact that the mere presence of a mother can be comforting to a child could be of value for families during a NICU stay. Furthermore, Sarro et al. found that maternal behaviours such as grooming and nurturing modulated infant cortical activity, sometimes inducing desynchronisation and sometimes reducing it. According to Sarro et al., there 'may be a mechanism allowing variation in maternal care to create variability in brain development and behavioural outcome. The nature and duration of specific maternal behaviours, as well as the temporal patterning of interaction and absence, could induce robust individual differences in arousal and consolidation, as well as network maturation'.

In a study on human subjects, Hane et al. (46) confirmed altered cortical activity due to differences in caregiving. They scored mothers according to variations in their maternal caregiving behaviour when their infants were nine months old. A correlation between these scores and infant EEG at three years was then investigated as an indicator of stress reactivity in human infants. When compared to high-quality maternal caregiving, low-quality maternal caregiving led to an increase in stress reactivity determined by right frontal EEG asymmetry.

In their booklet, Browne et al. described that the establishment of adequate bonding through such highquality caregiving has profound effects on animals' emotions and responses to stress, accompanied by important changes in their central levels of BDNF. As previously mentioned, BDNF is a regulator of neuronal development. Increased BDNF promotes and accelerates central nervous system maturation, possibly leading to more adaptive coping styles and reduced vulnerability to psychopathology (13). Browne et al. stated that 'researchers have therefore sought for ways to increase cerebral BDNF endogenously or exogenously in humans'. They concluded that the most feasible option to increase BDNF, as well as other substances that stimulate development, would be endogenously. Interventions that enrich the early life environment by focusing more on parent-infant bonding could as a result cause an endogenous increase in BDNF, as well as an increase in other substances important to development (13). 


\section{Human studies that assessed caregiving or interventions that increased bonding}

The most common example of a caregiving method that increases the focus on bonding in NICUs is Kangaroo care (KC). KC is a method of creating skin-to-skin contact by holding a baby in a supine position on a naked caregiver's chest when the baby is wearing nothing but a diaper (47). A longitudinal study by Feldman et al. was set up to examine the effect of $\mathrm{KC}$ on preterm infants, parent-infant interaction and child development (4). Infants who received KC for 14 consecutive days were matched to control infants, with both groups containing 73 infants. They were all assessed at three time intervals during the first half year and seven other time intervals during the first decade. During the first half year, family interaction and home environments were more adapted in the KC group. Mothers reported less depression and infants scored higher on the Bayley Mental Developmental Index and the Psychomotor Developmental Index. Feldman et al. speculated that KC had both a direct impact on the infants' neurophysiological regulation as well as an indirect impact by improving parental mood, perceptions and interactive behaviour (4). During the infants' first decade, KC increased maternal attachment behaviour and infants' autonomic functioning as measured by respiratory sinus arrhythmia. Furthermore, child cognitive development and executive functions were enhanced. By 10 years of age, KC children showed attenuated stress responses, more mature respiratory sinus arrhythmia patterns, better organised sleep and better cognitive control. Respiratory sinus arrhythmia patterns and maternal behaviour were dynamically inter-related over time, demonstrating coregulation (4). Such positive effects of $\mathrm{KC}$ were reinforced in a Cochrane review on Kangaroo mother care. This term is used to describe KC within a broader caregiving principle that adds almost exclusive breastfeeding and attempted early discharge from the hospital to daily, preferably long, KC sessions (47).

Other interventions aimed at increasing bonding described in the literature were, for instance, maternal voice recordings or maternal biological sounds (48) music therapy (49) or a breathing bear (50). Lahav et al. performed a number of studies on using maternal sounds in the NICU, all with promising effects. For example, in one study increased cardiorespiratory stability, with a reduced frequency of apnoea and bradycardia, was reported (48). Research, therefore, appears to support the use of familiar or positive sounds in NICUs. This was also confirmed by a recent meta-analysis on music therapy that concluded that evidence-based NICU music therapy was highly beneficial with an overall large significant effect size. Benefits were greatest with live music therapy, such as mothers singing (49). Voice vibrations and the rhythmicity of a maternal voice, as well as maternal breathing patterns and heart beats, are features that healthy, term born neonates normally rely upon to support their regulatory development (48). Access to such audible features could thus be of even more importance to preterm infants, who, as previously stated, are exposed to many unnatural stimuli and have a far more vulnerable physique (10). Breathing is a serious challenge for many preterm infants due to lung immaturity (1). Extra coregulatory support for breathing using audible biological sounds or music with vital rhythms could thus be very beneficial (48).

Thoman et al. used breathing sounds in a device suitable for preterm and full-term infants, called the breathing bear. This is a teddy bear designed to permit entrainment of the infant's breathing capacity and related neural functions. It breathes quietly to ensure that the intervention is not imposed on the infant. In a study with irritable full-term infants, the device was placed in the crib and the infant could control whether, when and how long to make contact with it and experience its rhythmic body motion. Compared to a control group of irritable infants without exposure to the breathing bear, positive effects on contact behaviour, temperament, maternal stress and maternal depression were reported (50). Superior effects to those of the breathing bear could presumably be caused by sleeping in the same bed as a sibling or parent.

Cobedding was investigated in a Cochrane review (51). Only five studies could be included in that review, all of which were small and reported their outcomes differently. There was, therefore, insufficient evidence to make recommendations for future practice. No significant differences in weight gain, apnoea, bradycardia, desaturation, infection incidence, length of hospital stay or parental perceptions were found. Cobedded twins appeared to spend more time crying, but they also appeared to spend more time in quiet sleep. Morgan et al. (52) examined whether infants should be cobedding with their mothers. In a within-subject design, they measured heart rate variability to assess autonomic function in 16, two-day-old full-term healthy neonates sleeping in skin-to-skin contact with their mothers and sleeping alone prior to discharge. All neonates were admitted to the hospital because they were born by Caesarean section. Both sleeping methods were measured for one hour. Results show a $176 \%$ increase in autonomic activity and a $86 \%$ decrease in quiet sleep duration during maternal separation compared to skin-to-skin contact. The separation thus appeared to increase stress.

Flacking et al. (23) reviewed the concept of closeness and separation in neonatal intensive care units more generally than the cobedding concept. They too highlighted the need for acknowledging the importance and impact of both physical and emotional closeness between the preterm infant and parent in the NICU. Emotional closeness referred to parental feelings of being emotionally connected, feelings of love, warmth and affection. In their review, the evidence that illustrated the importance of closeness came from stress and cortisol studies. Close physical contact between a parent and a preterm infant decreased the infant's cortisol levels and pain responses (53). In addition, an increase in the amount of physical contact and family-centred care synchronised cortisol variation between the preterm infant and his or her mother. 
It appeared that sharing the same environment increased the concordance between the HPA axis reactivity of the preterm infants and their mothers, reflected by the cortisol levels in their saliva (53). In addition, various interventions that have supported the abilities of parents to observe and interpret their infant's behaviour have all been associated with improved cognition years later $(4,54)$. Flacking et al. thus concluded that parent-infant closeness should be an absolute priority within NICUs (23).

The importance of closeness was also described in the Postnatal maternal care, tactile stimulation and brain development section in the review by Samra et al. Concerning that, they reported that preterm infants were vulnerable to programmed changes if their care environments were less than optimal. Furthermore, they reported on evidence linking prematurity and family-centred care to epigenetic modifications. They concluded that more research is needed to understand these variables and their moderating or mediating relationships to each other (55). An attempt to address this was made by Milgrom et al. and Welch et al. in their studies, which examined caregiving environments by investigating different interventions that supported parents.

With their early sensitivity training programme, Milgrom et al. (56) reached a significantly enhanced white matter maturation and connectivity on magnetic resonance imaging in prematurely born infants when they were 3840 weeks of age. Absolute volumes of cerebral tissue types revealed no differences. In this randomised, controlled trial, 45 women with infants younger than 30 weeks of gestation were involved. The intervention $(n=22)$ consisted of 10 sessions of parents working with therapists to learn to recognise signs of infant stress, shutdown mechanisms, alert available behaviour, quality of motor behaviours, facial expressions and posture or muscle tone. In comparison with NIDCAP, this intervention had relatively low costs due to less nurse training and nurse time assessing the infant. The parents assessed the preterm infant, which had possible postdischarge benefits as well. In addition to the increased white matter maturation, other medical outcomes demonstrated a general improvement for children in the intervention group as well. For example, fewer days on oxygen and a decreased length in hospital stay were reported. So, even though not all of these improvements were statistically significant, the findings of this study concurred with recent evidence that the quality of early life experiences influences physiological development and especially brain development. The results provided a platform for consideration of larger randomised trials investigating this type of interventions in preterm infants (56).

Welch et al. conducted such a large, randomised controlled trial that investigated an intervention with a different approach. The intervention developed and studied by the authors was called the Family Nurture Intervention. In their research, they assessed the impact of that intervention on EEG activity in 134 preterm infants born between 2634 weeks of gestation (57). The distinguishing feature of this intervention was that the staff facilitated communication on affect and physiological coregulation between the mother and her infant. The intervention started early and continued over the full course of hospitalisation. It entailed mother and infant scent cloth exchange, sustained touch, vocal soothing, eye contact, wrapped or skin-to-skin holding and family-based support sessions (58). Family Nurture Intervention infants showed robust increases in frontal brain activity on EEG, which other investigators found predictive of better neurobehavioural outcomes. Effects were significant in both quiet and active sleep, regardless of gender, gestational age or birth weight. Significant groupby-age effects in other brain regions were found as well (57). Additional studies assessing this intervention demonstrated not only improved maternal caregiving behaviour (59), but also significantly improved scores across multiple domains of neurodevelopment, social relatedness and attention problems in the intervention group compared to the control group receiving standard care (60). These results suggest widespread changes in developmental trajectories mediated by the Family Nurture Intervention, an intervention focused entirely on enhanced coregulation, or in other words, enhanced bonding.

\section{DISCUSSION}

Recent findings suggest that early life conditions turn out as either harmful or protective, depending on the organism's later life environmental context. This implies that early life conditions may prepare the infant for their future life through glucocorticoid programming and phenotypic plasticity with the goal to match with future environmental demands $(10,39,40)$. This concept has led to the hypothesis that a mismatch between early and later life conditions can enhance vulnerability to disease. For example, although the offspring of low maternal care dams seemingly displayed lower cognitive performance under basal conditions when compared to the offspring of high maternal care dams, a different outcome was described when the rats were tested under stressful conditions, that is a situation reminiscent of their early life environment $(28,33)$. These findings suggest that what used to be perceived as a maladaptive phenotype may turn out to represent an adaptation to the environmental context that prevailed during development, which appropriately predicts and matches with the environment later in life. This is a phenomenon that has adaptive values in particular contexts, but can be maladaptive when mismatched and persistent $(34,37,40)$.

More evidence for this theory comes from considering the correlations between the reproductive system and human parental programming over an evolutionary time span. Under high-risk conditions, when the probability of survival is low, the optimal strategy is to maximise the number of offspring through accelerated mating. In contrast, a more propitious environment favours greater investment in individual offspring at the cost of mating (43). Thus, the adaptive maturation of neural and endocrine systems requires environmental input to optimise their 
functions. The outcome is somewhere on the continuum between resilience and psychopathology. It depends on the nature of the input, the balance between stimulant as well as challenging input, the caregiving during the challenges, the respective genetic predispositions and on the social context in later life, which all depend on each other as well (28). Due to this complexity, the directions of changes are sometimes inconsistent and unexpected and even small differences can affect the direction of change. Additionally, changes can even be different within one brain region (39).

This review was not intended to explain those varying, context-dependent directions of alterations. It meant to raise clinicians' awareness of the omnipresence of changes caused by differences in bonding-related early life experiences, by providing a large, convincing amount of varying examples. Unfortunately, due to that variety, the actual summing up of findings, which would have resulted in a more number-oriented review, appeared impossible. Another limitation was the fact that the majority of this review consisted of animal studies. However, there is now strong evidence that human and animal parenting share many subcortical, neural and neurochemical mechanisms $(8-10,55)$. Furthermore, albeit not always significant, numerous positive effects of interventions that aim to optimise bonding in human studies have been reported, in contrast to not one negative effect. Improving bonding should therefore be prioritised $(10,13,60)$, particularly in high incidence, vulnerable populations such as preterm infants (1).

\section{CONCLUSION}

This review emphasises the importance of bonding by providing sound evidence for the fact that differences in bonding-related early life experiences and NICU-related early life experiences have a significant impact on physiological development in various ways. That impact is highly context dependent and frequently gender dependent as well. Furthermore, the impact can be reversed by improving the caregiving context and by optimising parent-infant bonding. The attention for adequate bonding in NICUs should therefore be increased.

\section{CONFLICT OF INTEREST}

None declared.

\section{ACKNOWLEDGEMENTS}

None.

\section{References}

1. Marcos Z. Arriving too early. Lancet Neurol 2013; 12: 332-3.

2. Aarnoudse-Moens CSH, Weisglas-Kuperus N, van Goudoever JB, Oosterlaan J. Meta-analysis of neurobehavioural outcomes in very preterm and/or very low birth weight children. Pediatrics 2009; 124: 717-28.
3. Grunau RE. Neonatal pain in very preterm infants: long-term effects on brain, neurodevelopment and pain reactivity. Rambam Maimonides Med J. 2013; 4: e0025.

4. Feldman R, Rosenthal Z, Eidelman AI. Maternal-Preterm Skinto-Skin Contact Enhances Child Physiologic Organization and Cognitive Control Across the First 10 Years of Life. Biol Psychiatry 2014; 75: 56-64.

5. Rinaman L, Banihashemi L, Koehnle TJ. Early life experience shapes the functional organization of stress-responsive visceral circuits. Physiol Behav 2011; 104: 632-40.

6. Tang AC, Reeb-Sutherland BC, Romeo RD, McEwen BS. On the causes of early life experience effects: Evaluating the role of mom. Front Neuroendocrinol 2014; 35: 245-51.

7. Bretherton I. The origins of attachment theory: John Bowlby and Mary Ainsworth. Dev Psychol 1992; 28: 759-75.

8. Mercer J. Attachment theory and its vicissitudes: toward an updated theory. Theory Psychol 2011; 21: 25-45.

9. Hofer MA. Psychobiological roots of early attachment. Curr Dir Psychol Sci 2006; 15: 84-8.

10. Feldman R. Parent-infant synchrony and the construction of shared timing; physiological precursors, developmental outcomes, and risk conditions. J Child Psychol Psychiatry Allied Discip 2007; 48: 329-54.

11. Fleming AS, O'Day DH, Kraemer GW. Neurobiology of mother-infant interactions: experience and central nervous system plasticity across development and generations. Neurosci Biobehav Rev 1999; 23: 673-85.

12. Carter CS. Oxytocin pathways and the evolution of human behaviour. Annu Rev Psychol 2014; 65: 17-39.

13. Browne JV, White RD. Clinics in Perinatology - Foundations in Developmental Care. Clin Perinatol 2011; 38: 591-758.

14. Als H. Developmental care in the newborn intensive care unit. Curr Opin Pediatr 1998; 10: 138-42.

15. Picther JB, Schneider LA, Drysdale JL, Ridding MC, Owens JA. Motor system development of the preterm and low birthweight infant. Clin Perinatol 2011; 38: 605-25.

16. Brummelte S, Grunau RE, Chau V, Poskitt KJ, Brant R, Vinall $\mathrm{J}$, et al. Procedural pain and brain development in premature newborns. Ann Neurol 2012; 71: 385-96.

17. Doesburg SM, Chau CM, Cheung TPL, Moiseev A, Ribary U, Herdman AT, et al. Neonatal pain-related stress, functional cortical activity and visual-perceptual abilities in school-age children born at extremely low gestational age. Pain 2013; 154 : 1946-52.

18. Anand KJS, Scalzo F. Can adverse neonatal experiences alter brain development and subsequent behaviour? Neonatology 2000; 77: 69-82.

19. Anand KJS, Garg S, Rovnaghi CR, Narsinghani U, Bhutta AT, Hall RW. Ketamine reduces the cell death following inflammatory pain in newborn rat brain. Pediatr Res 2007; 62: 283-90.

20. Song J, Lee JH, Lee SH, Park KA, Lee WT, Lee JE. TRPV1 Activation in Primary Cortical Neurons Induces CalciumDependent Programmed Cell Death. Exp Neurobiol 2013; 22: $51-7$.

21. Ohlsson A, Jacobs SE. NIDCAP: a systematic review and metaanalyses of randomized controlled trials. Pediatrics 2013; 131: e881-93.

22. Ohlsson A, Jacobs SE. Authors' response: NIDCAP: a Systematic Review and Meta-Analyses of Randomized Controlled Trials. Pediatrics 2013; 132: e553-7.

23. Flacking R, Lehtonen L, Thomson G, Axelin A, Ahlqvist S, Moran $\mathrm{VH}$, et al. Closeness and separation in neonatal intensive care. Acta Paediatr 2012; 101: 1032-7.

24. Latva R, Lehtonen L, Salmelin RK, Tamminen T. Visiting less than every day: a marker for later behavioural problems in 
Finnish preterm infants. Arch Pediatr Adolesc Med 2004; 158: 1153-7.

25. Sullivan R, Perry R, Sloan A, Kleinhaus K, Burtchen N. Infant bonding and attachment to the caregiver: insights from basic and clinical science. Clin Perinatol 2011; 38: 643-55.

26. Walker CD. Maternal touch and feed as critical regulators of behavioural and stress responses in the offspring. Dev Psychobiol 2010; 52: 638-50.

27. Ziabreva I, Schnabel R, Braun K. Parental deprivation induces $\mathrm{N}$-methyl-D-aspartate-receptor upregulation in limbic brain areas of Octodon degus: protective role of the maternal call. Neural Plast 2000; 7: 233-44.

28. Bock J, Rether K, Gröger N, Xie L, Braun K. Perinatal programming of emotional brain circuits: an integrative view from systems to molecules. Front Neurosci 2014; 8: 11.

29. Levine S. Regulation of the hypothalamic-pituitary-adrenal axis in the neonatal rat: the role of maternal behaviour. Neurotox Res 2002; 4: 557-64.

30. Rincón-Cortés M, Sullivan RM. Early life trauma and attachment: immediate and enduring effects on neurobehavioural and stress axis development. Front Endocrinol 2014; 5: 33.

31. Liu D, Diorio J, Tannenbaum B, Caldji C, Francis D, Freedman A, et al. Maternal Care, Hippocampal Glucocorticoid Receptors, and Hypothalamic-Pituitary-Adrenal Responses to Stress. Science 1997; 277: 1659-62.

32. Hostinar CE, Sullivan RM, Gunnar MR. Psychobiological mechanisms underlying the social buffering of the hypothalamic-pituitary-adrenocortical axis: a review of animal models and human studies across development. Psychol Bull 2014; 140: 256-82.

33. Strüber N, Strüber D, Roth G. Impact of early adversity on glucocorticoid regulation and later mental disorders. Neurosci Biobehav Rev 2014; 38: 17-37.

34. Letourneau N, Giesbrecht GF, Bernier FP, Joschko J. How do interactions between early caregiving environment and genes influence health and behaviour? Biol Res Nurs 2014; 16: 83-94.

35. Kundakovic M, Champagne FA. Early-life experience, epigenetics, and the developing brain. Neuropsychopharmacology 2014. doi:10.1038/npp. 2014.140

36. Meaney MJ, Szyf M, Seckl JR. Epigenetic mechanisms of perinatal programming of hypothalamic-pituitary-adrenal function and health. Trends Mol Med 2007; 13: 269-77.

37. Weaver ICG. Toward an Understanding of the Dynamic Interdependence of Genes and Environment in the Regulation of Phenotype. In: A Petronis, J Mill, editors. Brain, Behaviour and Epigenetics. Berlin: Springer-Verlag, 2011: 209-43.

38. Murgatroyd C, Patchev AV, Wu Y, Micale V, Bockmühl Y, Fischer D, et al. Dynamic DNA methylation programs persistent adverse effects of early-life stress. Nat Neurosci 2009; 12: 1559-66.

39. Veenema AH. Toward understanding how early-life social experiences alter oxytocin- and vasopressin-regulated social behaviours. Horm Behav 2012; 61: 304-12.

40. Buisman-Pijlman FTA, Sumracki NM, Gordon JJ, Hull PR, Carter CS, Tops M. Individual differences underlying susceptibility to addiction: role for the endogenous oxytocin system. Pharmacol Biochem Behav 2013; 119: 22-38.

41. Todeschin AS, Winkelmann-Duarte EC, Jacob MHV, Aranda BCC, Jacobs S, Fernandes MC, et al. Effects of neonatal handling on social memory, social interaction, and number of oxytocin and vasopressin neurons in rats. Horm Behav 2009; 56: $93-100$
42. Peña CJ, Neugut YD, Calarco CA, Champagne FA. Effects of maternal care on the development of midbrain dopamine pathways and reward-directed behaviour in female offspring. Eur J Neurosci 2014; 39: 946-56.

43. Toufexis D, Rivarola MA, Lara H, Viau V. Stress and the Reproductive Axis. J Neuroendocrinol 2014; 26: 573-86.

44. Camozzato TSC, Winkelmann-Duarte EC, Padilha CB, Miguel SPR, Bonzanini L, Anselmo-Franci JA, et al. Neonatal handling reduces the number of cells in the medial preoptic area of female rats. Brain Res 2009; 1247: 92-9.

45. Sarro EC, Wilson DA, Sullivan RM. Maternal Regulation of Infant Brain State. Curr Biol 2014; 24: 1664-9.

46. Hane AA, Henderson HA, Reeb-Sutherland BC, Fox NA. Ordinary variations in human maternal caregiving in infancy and biobehavioural development in early childhood: a followup study. Dev Psychobiol 2010; 52: 558-67.

47. Conde-Agudelo A, Diaz-Rossello JL. Kangaroo mother care to reduce morbidity and mortality in low birthweight infants (Review). Cochrane Database Syst Rev 2014; 4: 1-65.

48. Doheny L, Hurwitz S, Insoft R, Ringer S, Lahav A. Exposure to biological maternal sounds improves cardiorespiratory regulation in extremely preterm infants. J Matern Fetal Neonatal Med 2012; 25: 1591-4.

49. Standley J. Music therapy research in the NICU: An updated meta-analysis. Neonatal Netw 2012; 31: 311-6.

50. Novosad C, Thoman EB. The breathing bear: an intervention for crying babies and their mothers. J Dev Behav Pediatr 2003; 24: 89-95.

51. Lai NM, Foong SC, Foong WC, Tan K. Co-bedding in neonatal nursery for promoting growth and neurodevelopment in stable preterm twins. Cochrane Database Syst Rev 2012; 12: CD008313.

52. Morgan BE, Horn AR, Bergman NJ. Should neonates sleep alone? Biol Psychiatry 2011; 70: 817-25.

53. Mörelius E, Örtenstrand A, Theodorsson E, Frostell A. A randomised trial of continuous skin-to-skin contact after preterm birth and the effects on salivary cortisol, parental stress, depression and breastfeeding. Early Hum Dev 2015; 91 : 63-70.

54. Melnyk BM, Feinstein NF, Alpert-Gillis L, Fairbanks E, Crean HF, Sinkin RA, et al. Reducing premature infants' length of stay and improving parents' mental health outcomes with the Creating Opportunities for Parent Empowerment (COPE) neonatal intensive care unit program: a randomized, controlled trial. Pediatrics 2006; 118: e1414-27.

55. Samra HA, McGrath JM, Wehbe M, Clapper J. Epigenetics and family-centered developmental care for the preterm infant. Adv Neonatal Care 2012; 12(Suppl. 5): S2-9.

56. Milgrom J, Newnham C, Anderson PJ, Doyle LW, Gemmill $\mathrm{AW}$, Lee $\mathrm{K}$, et al. Early sensitivity training for parents of preterm infants: impact on the developing brain. Pediatr Res 2010; 67: 330-5.

57. Welch MG, Myers MM, Grieve PG, Isler JR, Fifer WP, Sahni R, et al. Electroencephalographic activity of preterm infants is increased by Family Nurture Intervention: a randomized controlled trial in the NICU. Clin Neurophysiol 2013; 125: 0-9.

58. Welch MG, Hofer MA, Brunelli SA, Stark RI, Andrews HF, Austin J, et al. Family Nurture Intervention (FNI): methods and treatment protocol of a randomized controlled trial in the NICU. BMC Pediatr 2012; 12: 1-17.

59. Hane AA, Myers MM, Hofer MA, Ludwig RJ, Halperin MS, Austin J, et al. Family Nurture Intervention Improves the Quality of Maternal Caregiving in the Neonatal Intensive Care Unit: evidence from a Randomized Controlled Trial. J Dev Behav Pediatr 2015; 36: 188-96. 
60. Welch MG, Firestein MR, Austin J, Hane AA, Stark RI, Hofer MA, et al. Family Nurture Intervention in the Neonatal Intensive Care Unit improves social-relatedness, attention, and neurodevelopment of preterm infants at 18 months in a randomized controlled trial. J Child Psychol Psychiatry 2015. doi:10.1111/jcpp.12405.

\section{APPENDIX: METHODS}

A MEDLINE and EMBASE search with broad search terms was undertaken through May-October 2014, to extract bonding-related perinatal factors from the available literature as a first step. There were four sorts of search terms; for the population (neonates, infants, etc.), for the determinant (bonding, caregiving, etc.) and for the changing (chang*, improv*, develop*) of the physiology with an emphasis on brain/neuronal physiology (brain, neuron*, EEG, physiology, organ, homeostas*, etc.; see Table A1). These search terms were decided upon in advance, based on their frequent usage in previously read, relevant articles. A thesaurus was used to find additional relevant terms. All matching titles were assessed and either included or excluded, according to predetermined, broad criteria (see Table A2). During the assessment of all the titles, terms used for bonding-related perinatal factors and frequently occurring outcome parameters were extracted. They were written down to find a structured answer to the question

Table A1 Search terms. At least one synonym of each term present in title/abstract.

\begin{tabular}{llll} 
Term 1 & Term 2 & Term 3 & Term 4 \\
\hline Develop* & Maternal care & Premature & Physiologic* \\
Improv* & Paternal care & Newborn* & Organ \\
Growth & Parental care & Childhood & Homeostas* \\
Chang* & Standard care & Infan* & Brain \\
Differentiat* & Caregiv* & Preterm & Neuron* \\
Matur* & Nurtur* & Perinatal & Hippocamp* \\
& Bonding & Postnatal & Axon* \\
& Attachment & Neonat* & EEG \\
& 'Mother infant' & Offspring & MRI \\
& & & Dendrit* \\
& & & Receptor* \\
& & &
\end{tabular}

*Means all possible extensions of the given word are included.

Table A2 Predetermined criteria for title inclusion or exclusion.

\section{Inclusion criteria} Exclusion criteria

All childhood experiences

All physiological outcomes
Article not in English, Dutch, French or German

Explicitly about children with syndromal diseases only Explicitly comparing only socio-economical influences (e.g. poverty, malnutrition) Explicitly concerning mothers with psychiatric diseases only 'what does suboptimal bonding do to physiological development'. With the written down terms, an associative and categorised framework was constructed (see Table A3), before reading the abstracts and full texts.

Successively, abstracts were screened to either subdivide them into their categories (preliminary inclusion) or to exclude them. The same broad inclusion and exclusion criteria that were previously used to screen the titles with were used for this abstract screening process (Table A2). After this screening process, the included abstracts and their full texts were read in order to include or exclude articles according to new, more specific inclusion and exclusion criteria (that were also predetermined). For these criteria, see Table A4. Some articles were redistributed. In addition, reference lists of identified articles and related reviews were hand searched. For the final flowchart of all included articles per topic, see Figure A1.

The subsequent writing stage consisted of three more steps. First, an extensive, non-selective work containing all

Table A3 Associative framework dividing all the articles into seven categories after inclusion.

Terms for early life bonding-related factors

Parental licking and grooming

Parental presence/separation

Pysiological outcome parameters

Environmental enrichment

Dendritic spine density

Kangaroo Mother Care

Institutionalisation

Attachment style

Received caregiving

Bonding

Feeding routines

Parental sensitivity

Tactile stimulation

Body heat

Early life experience

Early life adversity

Abuse

Neonatal handling

Still-face paradigm

Nurturing intervention

Comfort(ing)

Comfortin device

(High) quality caregiving

Developmental care

NICU standards/NICU care

Oxytocin

Cortisol

Altered amygdala appearance

Dopamine

HPA axis reactivity

Stress-axis functionality

Receptors

Synapses

Hormonal concentrations

Methylation

Epigenetic changes

Epigenetic modulations

Hippocampal changes

....many more...

Physiological outcome parameters divided into five categories

Stress-axis parameters

Genetic parameters

Hormonal parameters

Neuronal parameters

All outcomes

$\downarrow \downarrow \downarrow \downarrow \downarrow \downarrow \downarrow \downarrow \downarrow \downarrow \downarrow \downarrow \downarrow \downarrow$

Seven categories into which all included articles are divided

1 All factors concerning parental care altering stress-axis parameters

2 All factors concerning parental care altering genetic parameters

3 All factors concerning parental care altering hormonal parameters

4 All factors concerning parental care altering neuronal parameters

5 Caregiving in institutions or hospitals altering all physiological outcomes

6 Enriched environments altering all physiological outcomes

7 Bonding increasing interventions altering all physiological outcomes 


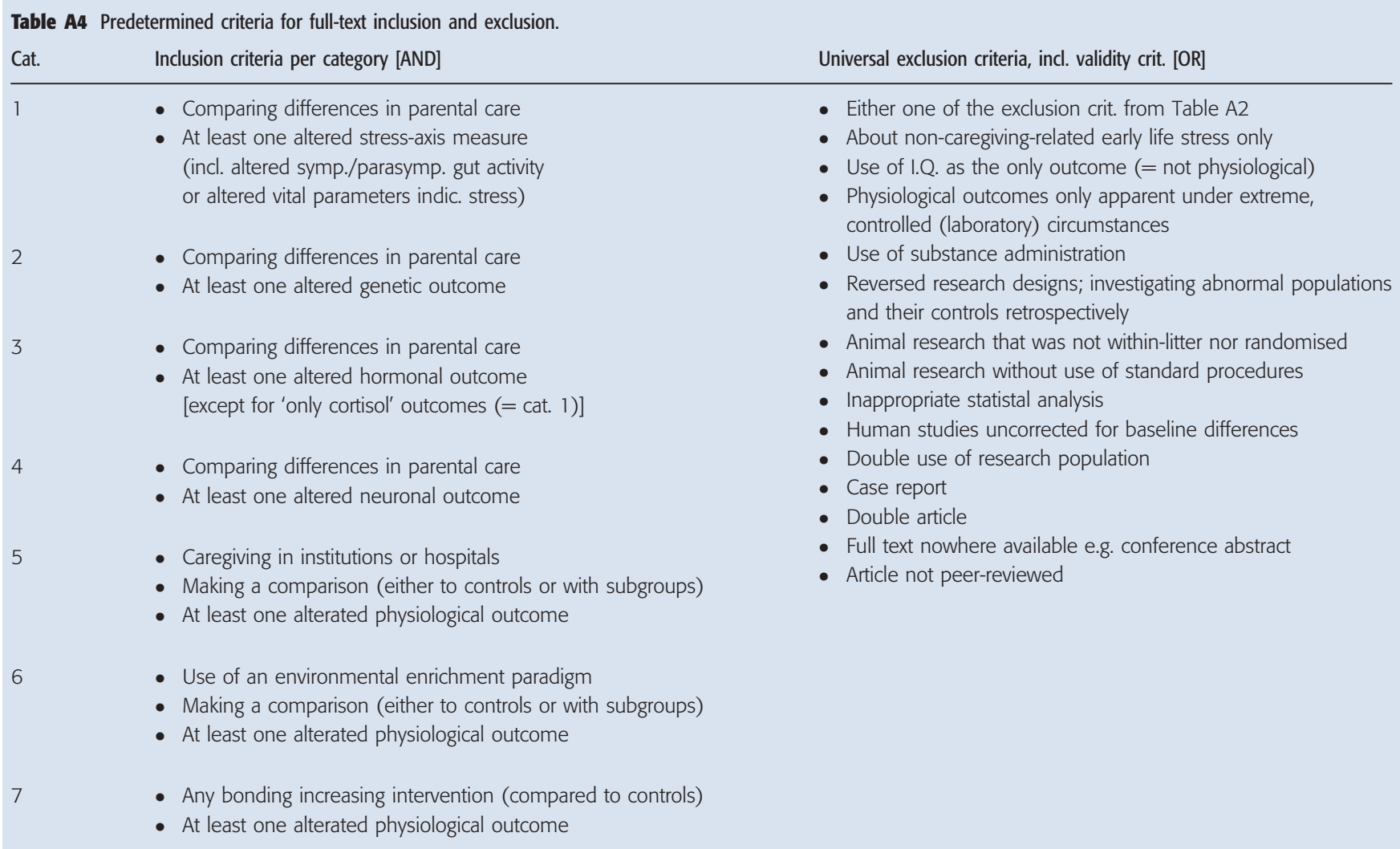

Cat, Category; incl, Including; crit, Criteria; symp./parasymp, Sympathetic/parasympathetic; indic, Indicating; I.Q, Intelligence quotient.

Table A5 Final filtering step; criteria to narrow down the results to be used in this review.

Inclusion criteria

Exclusion criteria

Clinically relevant

Directly linked to

the conclusion

Not importantly contributing to theoretical background Expected/similar finding to those previously described Too difficult to translate to human research

findings from all included articles per topic was created. Then, the general conclusion was written, based on undisputed issues only. This conclusion was now used as the final measurement for information to be valued with, to minimise the selection bias while narrowing down the article. Significance to that conclusion, as decided upon with some final predetermined criteria (Table A5), led to the final inclusion, exclusion and reordering of the findings. Various illustrating results that were exemplifying but did not independently add value to the conclusion were thus excluded.

During the revision phase, the main search was repeated and an extra hand search was executed in order to be as up to date, as explicit and as complete as possible. 


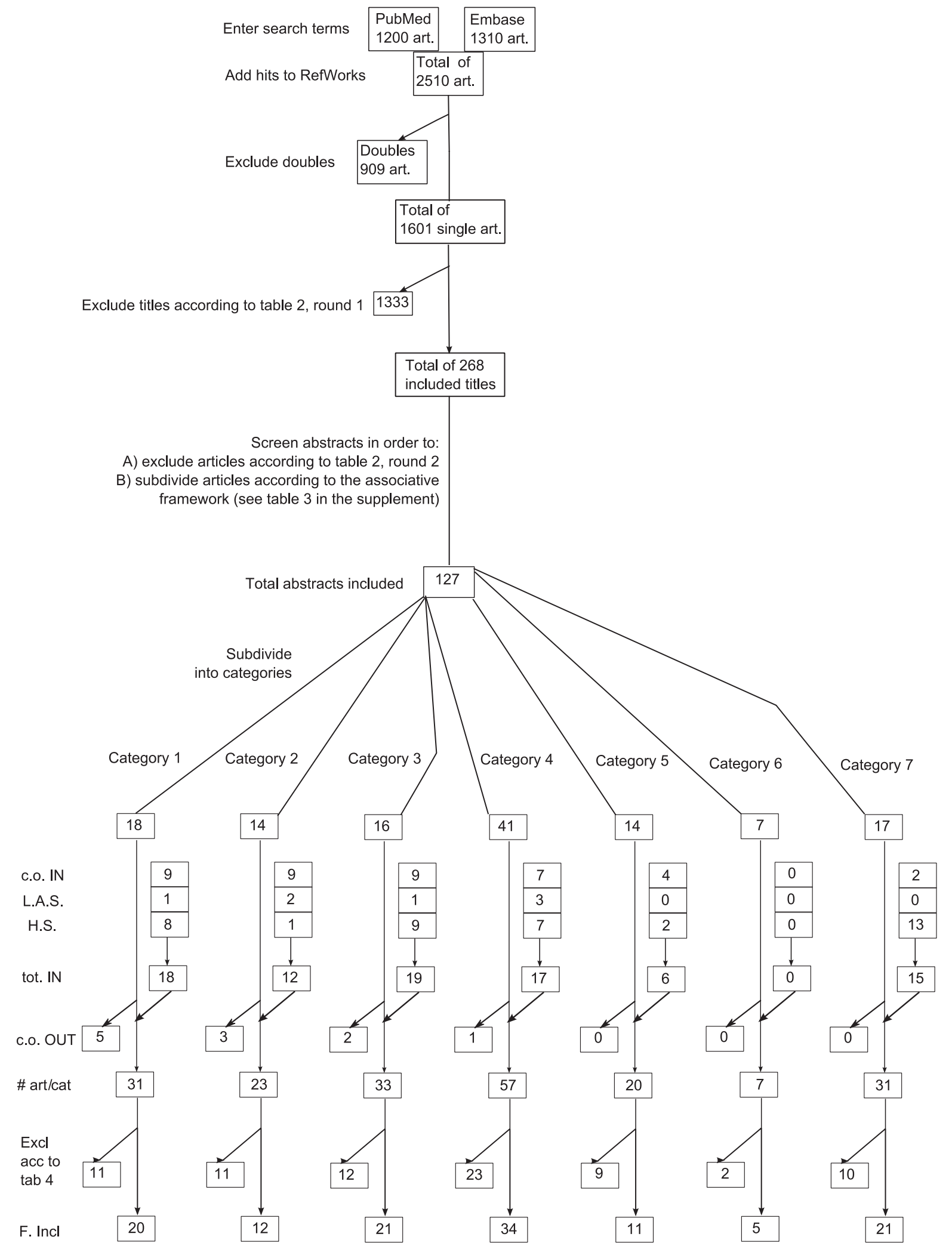

Fig. A1 Flow chart literature search. Art, articles; \# art/cat, current number of articles per category; c.o. IN, cross over ingoing, from another category into this category; L.A.S., later automatic searches, automatically done by PubMed and EMBASE; H.S., handsearch, articles included by reference guided handsearches; tot. IN, total amount of articles included; c.o. Out, cross-over Outgoing, from this category into another one; Excl acc. to tab 4, excluded according to Table A4; F. Incl, Final number of articles included. 